\title{
The Pressure Characteristics of the Released Gases from Sand Cores
}

\author{
L. MÁDI ${ }^{1}$, I. BUDAVÁRI $^{2}$, L. VARGA ${ }^{3}$ \\ 1,2,3University of Miskolc, Faculty of Materials Science and Engineering, Foundry, Department of Automotive \\ Castings, 1madi.laura.johanna@gmail.com. ${ }^{2}$ budavari1987@gmail.com, 3laszlo.varga.mak@gmail.com
}

Keywords: casting, sand cores, core gas, gas pressure, binders, resin content

Abstract. The core packages used for the production of castings are generally made from cores of different quality (no-bake phenol, HB-phenol, and HB-furan) and resin quantity, to meet the various requirements for the casting. In our research, the effect of the amount of resin on the pressure of the gases evolved from the cores was investigated. Experiments have shown that increasing the amount of resin has a different effect on different binder systems.

\section{Introduction}

The examination of the gas and its pressure generated from sand cores is a matter of priority in many respects. The core venting practices become increasingly more difficult with increased complexity and weight loss. On the other hand, the composition of the several gases evolved is an important environmental issue, as the regulations are getting stricter. When the initial contact between the metal and the mold is made, the temperature rises enormously, and the condensed water near the interface vaporizes, expanding in volume over 1000 times [1]. The relation of the speed of the released and removed gas determines the gas pressure in the core [2]. In that case, if the gas pressure exceeds the metallostatic head pressure, the gas would bubble through the molten aluminum. The intense gas release of the core can result in blowholes. Blowholes casting defects can be observed in Figure 1.

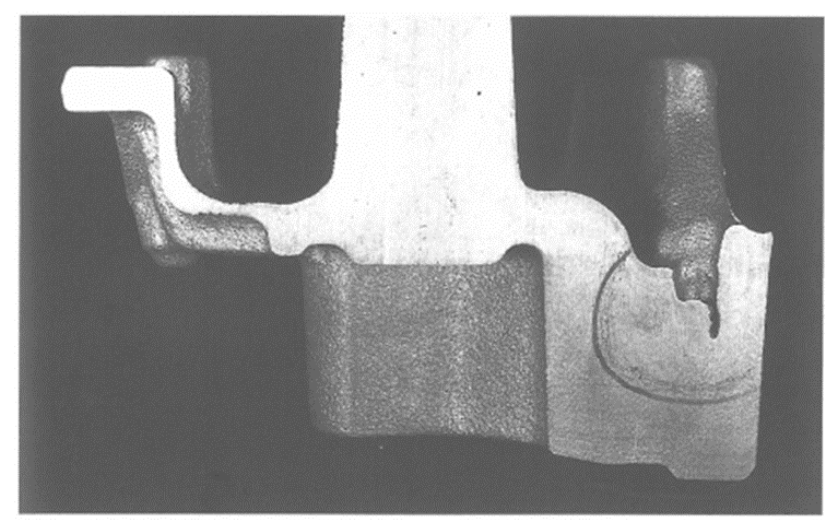

Figure 1. Blowholes casting defect in cast iron section [3]

Different pressures keep a balance at the metal/mold interface, such as metallostatic pressure, dynamic pressure, pressure during swelling of cast iron, capillary pressure, pressure drop due to 
friction and core gas pressure [4]. Core gas pressure is influenced by many factors, such as the resin content, the core permeability and the distance to core print. Higher pressure also occurs for cores with long distance to core prints, and for cores that decompose to produce higher viscosity gases. The gas evolution rate and viscosity increase with immersion temperature. The higher thermal conductivity of sand would allow the heat to penetrate quickly into the core and to develop higher gas pressure. Creasing resin content significantly increases the pressure. By increasing the permeability of the core, the internal gas pressure is characterized by an asymptotic decrease. Schematic diagram of the effect of the core permeability, the distance to core print and the resin content on core pressure can be observed in Figure 1.
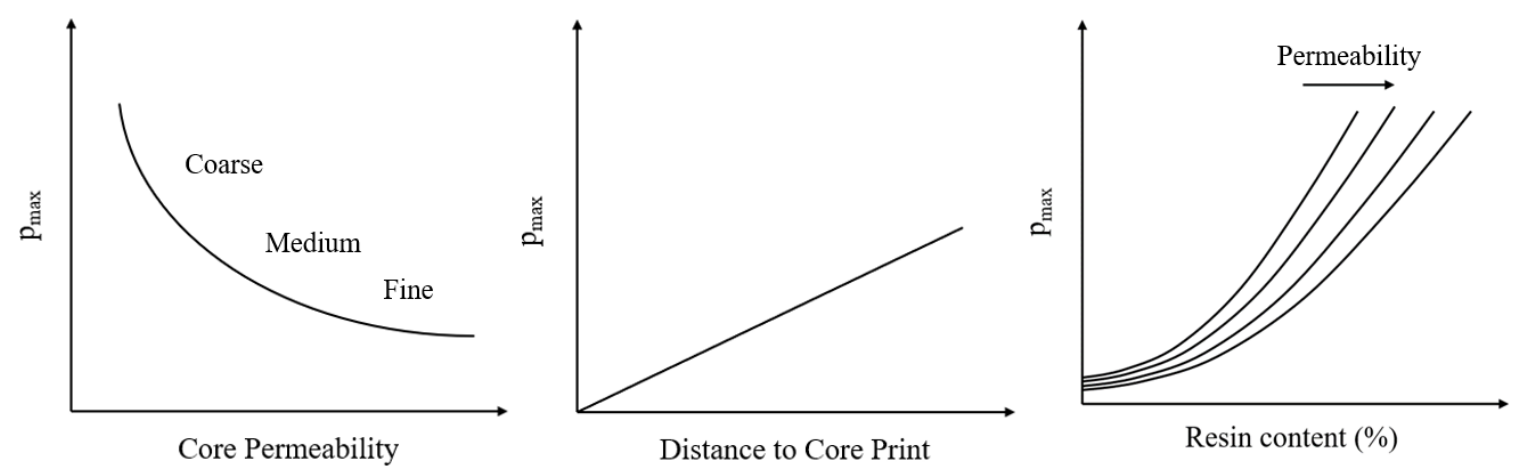

Figure 2. Schematic diagram of the effect of a) the core permeability b) the distance to core print c) the resin content on core pressure

The main topic of future research will focus on modeling gas evolution inside more complex commercial cores to predict the internal pressure and hence, the occurrence of gas defects. However, a more accurate model requires the type and concentration of gases evolved and more accurate interfacial heat transfer and apparent sand conductivity data. Knowledge of the type and concentration of the gases produced, the heat transfer between the surfaces and the apparent conductivity of the sand are essential for modeling $[5,6]$. The aim of this article is to investigate the impact of the quantity and quality of resin binder on the gas pressure of released gases from sand cores.

\section{Materials and method}

Synthetic organic resins used as foundry cores can cure by different chemistry and coremaking process, such as at room temperature (no-bake), in a hot-box (HB) or by gas curing processes (CB). The sand makes up at least $98-99+\%$ of the mixture for all heat-cured, no-bake, and cold-box binder systems used for the making of molds and cores. In the no-bake process, involving "cold" or room temperature curing with direct addition of a curing agent, cores and molds are produced from selfsetting sands in cold (ambient temperature of the foundry). In the hot-box coremaking technique, the wet sand mix is cured at $180-250^{\circ} \mathrm{C}$ in heated coreboxes. In "gas curing" processes, one reaction component is introduced into the sand mix in the form of a gas or vapor, which initiates the curing process. The types of resin and curing agent are selected to match the specific production conditions 
as well as the requirements of special cores. The test bars were prepared with various resin qualities (no-bake phenol, HB-phenol, and HB-furan) [7, 8].

Formaldehyde is added to phenol to form phenolic resin bonded molding and core sand mixtures. During the curing reaction, methylene is formed, which is capable of polymerization with water polycondensation. This process takes place in acidic conditions when organic acids are present such as para-toluenesulfonic acid (PTS) or benzenesulfonic acid (BSS). The phenolic resin may cure at room temperature or in a hot-box (about $220^{\circ} \mathrm{C}$ ). There is practically no nitrogen formation from this resin During the preparation of furan resin-bonded cores, the furfuryl alcohol crosslinks in an acidic medium to form a furan resin which, when cured, adheres to the sand particles. The curing process takes place either at room temperature (no-bake) or in a warm corebox $\left(200{ }^{\circ} \mathrm{C}\right)$. In both cases, the acidic medium may be an organic acid. Because of its nitrogen content, it is not suitable for steel casting purposes, as it can cause pinholes. Cold bonding is generally used to make larger molds and cores, and hot bonding is used to make smaller molds and cores. The mixing and metering of the various binder ingredients are critical in order to make high-quality castings. The molding sands are generally prepared by using the following composition as a guideline. The amount of binder used is usually $0.7-1.5 \%$, based on sand weight. Catalyst levels are generally-from 20 to $60 \%$, based on the weight of the binder. The binder content recommended by the manufacturer and cores containing $30 \%$ less and 30\% more binder is also examined. The composition of the sand mixtures is shown in Table 2. During sand mixture making, the acid catalyst is dispersed in the silica sand with a mixer and then the sand grains are coated with the liquid resin. The geometries of the test bars were $\emptyset 28 x 134,3$ $\mathrm{mm}$. The cores were prepared with constant compacting energy and approximately the same bulk density $\left(1.3 \mathrm{~g} / \mathrm{cm}^{3}\right)$.

\begin{tabular}{|c|c|c|c|c|}
\hline Resin quality & $\begin{array}{c}\text { Resin } \\
\text { content, } \\
\text { wt\% }\end{array}$ & $\begin{array}{c}\text { Catalyst } \\
\text { content, wt\% }\end{array}$ & $\begin{array}{c}\text { Curing } \\
\text { temperature, } \\
{ }^{\circ} \mathbf{C}\end{array}$ & $\begin{array}{c}\text { Curing } \\
\text { time, min }\end{array}$ \\
\hline Phenol no-bake & $1-1.2$ & $0.5-0.6$ & \multicolumn{2}{|c|}{ No-bake } \\
\hline HB-phenol & $1-1.5-2$ & $0.2-0.3-0.4$ & 220 & 15 \\
\hline HB-furan & $1-1.3-1.6$ & $0.25-0.33-0.41$ & 200 & 10 \\
\hline
\end{tabular}

Table 1. The composition of the sand mixtures

\section{Experimental procedures}

The measurement is similar to the actual production conditions. Stainless steel tube $(\varnothing 6 \times 300 \mathrm{~mm})$ were used as pressure probes; they were placed in the axial center of the core and so that their ends were $5 \mathrm{~mm}$ from the mold-metal interface, the closest distance at which the molten metal would not plug the tube. In the literature, the pressure of the gases was measured at a distance of $0.635 \mathrm{~cm}(0.25$ in.) from the mold-metal interface $[9,10]$.

The form was prepared using a green sand mixture containing $8 \%$ bentonite and $4 \%$ water. The fireresistant matrix of the form was silica sand. The drag box was $\emptyset 330 \times 200 \mathrm{~mm}$ and the cope box was $\emptyset 330 \times 140 \mathrm{~mm}$. The casting was carried out simultaneously using a tangential sprue in a three-cavity 
form. Approximately $50 \mathrm{~kg}$ green sand mixture was necessary for each form which was produced with a Multiserw Morek low-speed mixers. The mold cavities were made with a cone-shaped wood pattern $\left(\varnothing 70 \times 150 \mathrm{~mm}\right.$, draft: $\left.4^{\circ}\right)$. The cores with the steel tubes in the drag box were placed and poured around with liquid metal. The gating system was formed in the cope box. preparation steps of the sand mold can be observed in Figure 3.
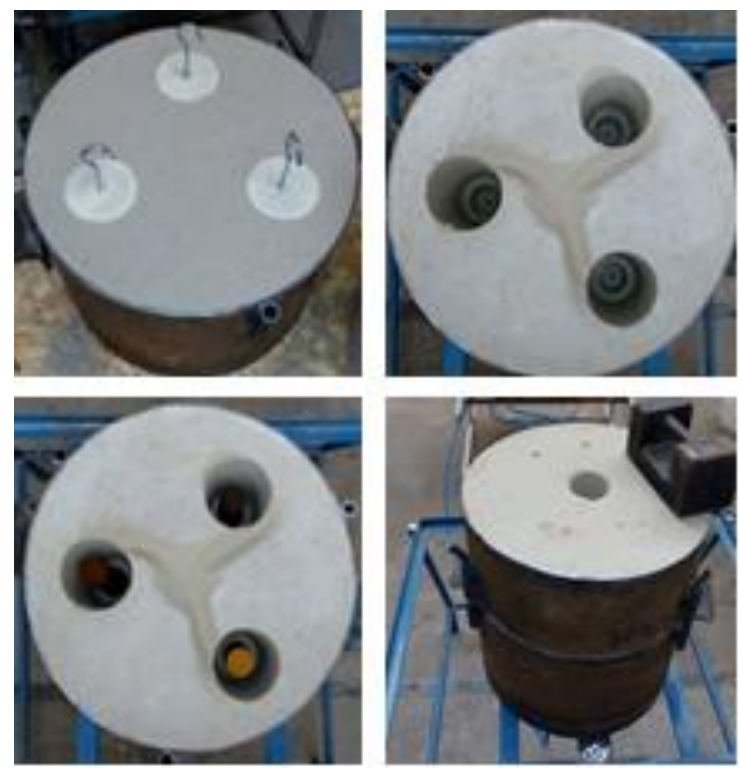

Figure 3. The experimental molding box, a) lower part with the cone-shaped wood pattern, b) tangential access channel without cores and c) with core, d) drag and cope box with weight on

$720^{\circ} \mathrm{C}$ foundry aluminum alloy was used for casting. CAD drawing of the three-core aluminum casting with the cores can be observed in Figure 4.
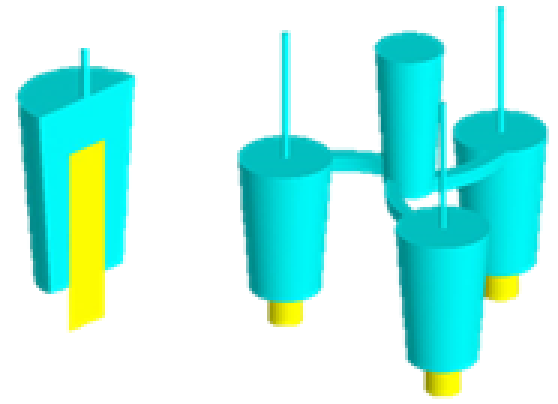

Figure 4. CAD drawing of the three-core aluminium casting with the cores a) section of the casting with cores b) cross-sectional view

The produced gases passed through a silicone tube to a pressure transducer. Three Baumer-type pressure transducers were used for the experiments.

\section{Experimental results}

The core packages used for the production of castings are generally made from cores of different quality (no-bake phenol, HB-phenol, and HB-furan) and resin quantity. In our research, the effect of 
the amount of resin on the pressure of the gases evolved from the cores was investigated. The effect of resin quality on the pressure of the released gases can be observed in Figure 5.

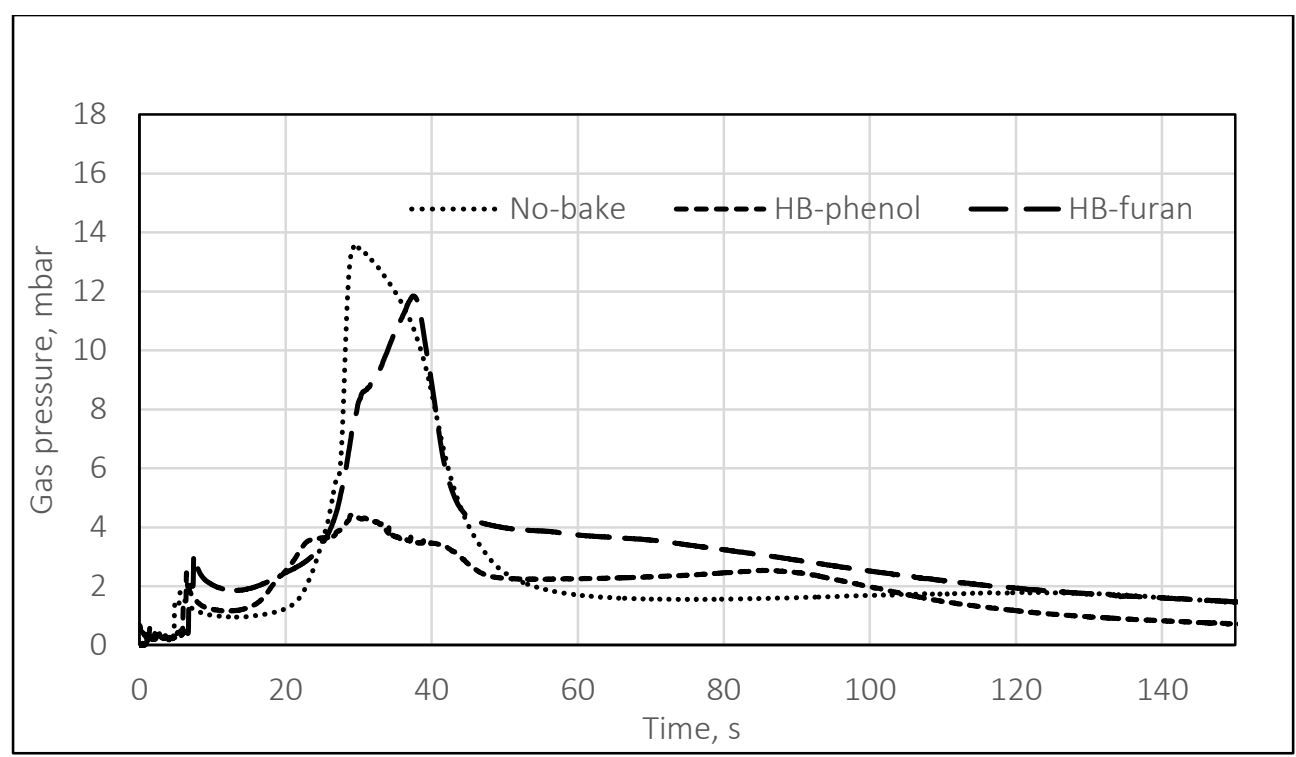

Figure 5. The effect of resin quality on the pressure of the released gases [11]

The measurements prove that the gas pressure curves are strongly influenced by the resin quality. The first pressure peak can be observed less than $10 \mathrm{~s}$ after casting, in case of $720^{\circ} \mathrm{C}$ casting temperature. The pressure peaks vary between 4-14 mbar in case of the different resin qualities [11].

\subsection{The effect of resin quantity on the pressure of the released gases}

During core making and casting, the composition of the sand cores may not be uniform, so it is also necessary to test the cores with different resin contents. In the case of HB-furan resin cores, the effect of different amounts of resin on the pressure of the gases evolved from the cores is shown in Figure 6.

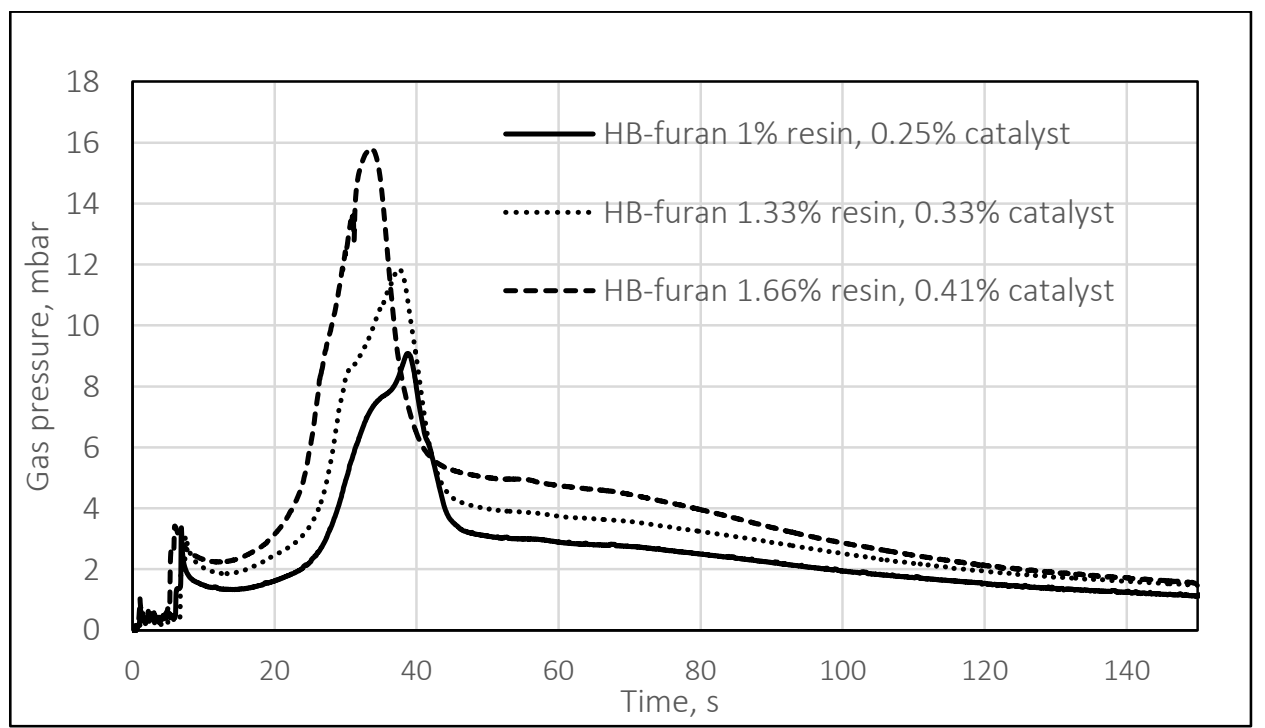

Figure 6. The effect of resin quantity on the pressure of the released gases in case of HB-furan 
As the resin content increases, core gas pressure increases significantly, and gas pressure peaks appear earlier in time. The pressure of gases released from HB-furan resin cores can be significantly influenced by the resin content. In the case of HB-phenol resin cores, the effect of different amounts of resin on the pressure of the gases evolved from the cores is shown in Figure 7.

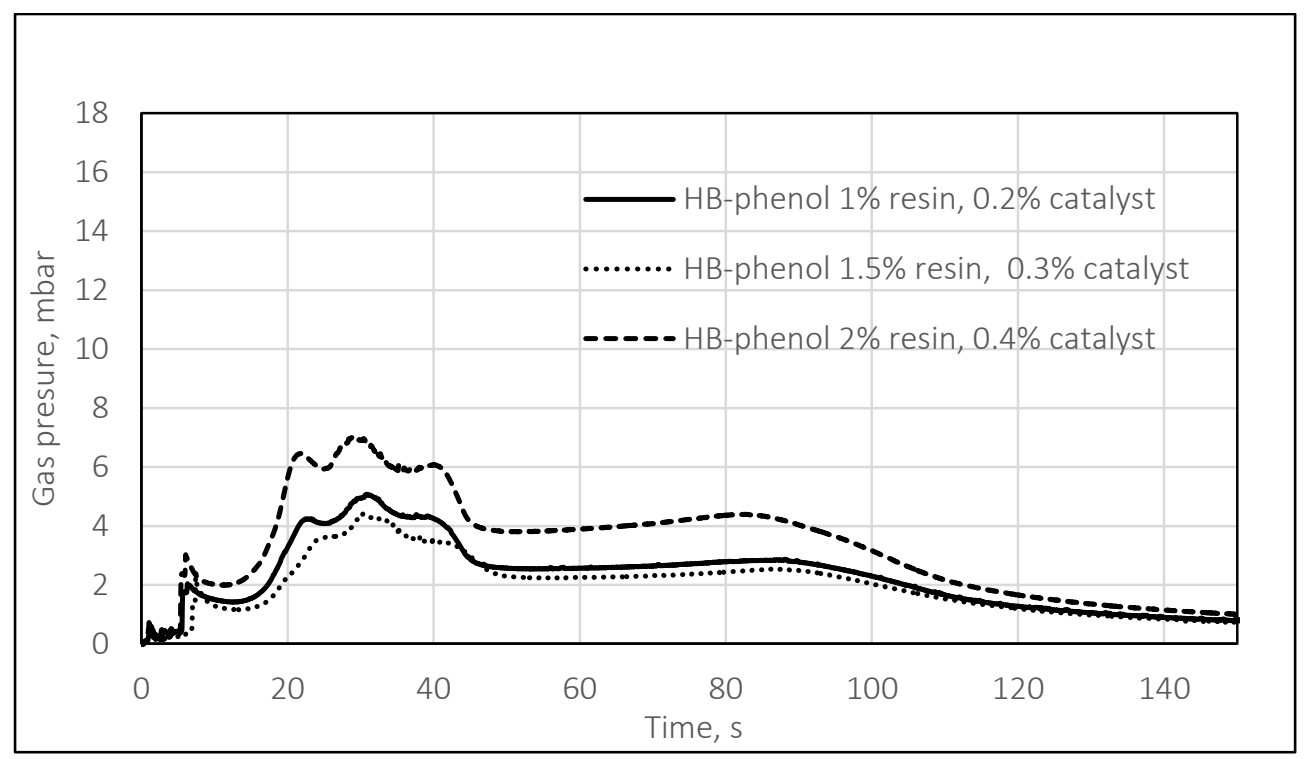

Figure 7. The effect of resin quantity on the pressure of the released gases in case of HB-phenol

In the case of HB-phenolic cores, the characteristics of the gas pressure curves are characterized by several local maximums and minimums, which can be explained by the intermittent resin burning. Increasing the resin content of HB-phenol resin cores does not cause a significant increase in pressure. In the case of no-bake phenol cores, the effect of different amounts of resin on the pressure of the gases evolved from the cores is shown in Figure 8.

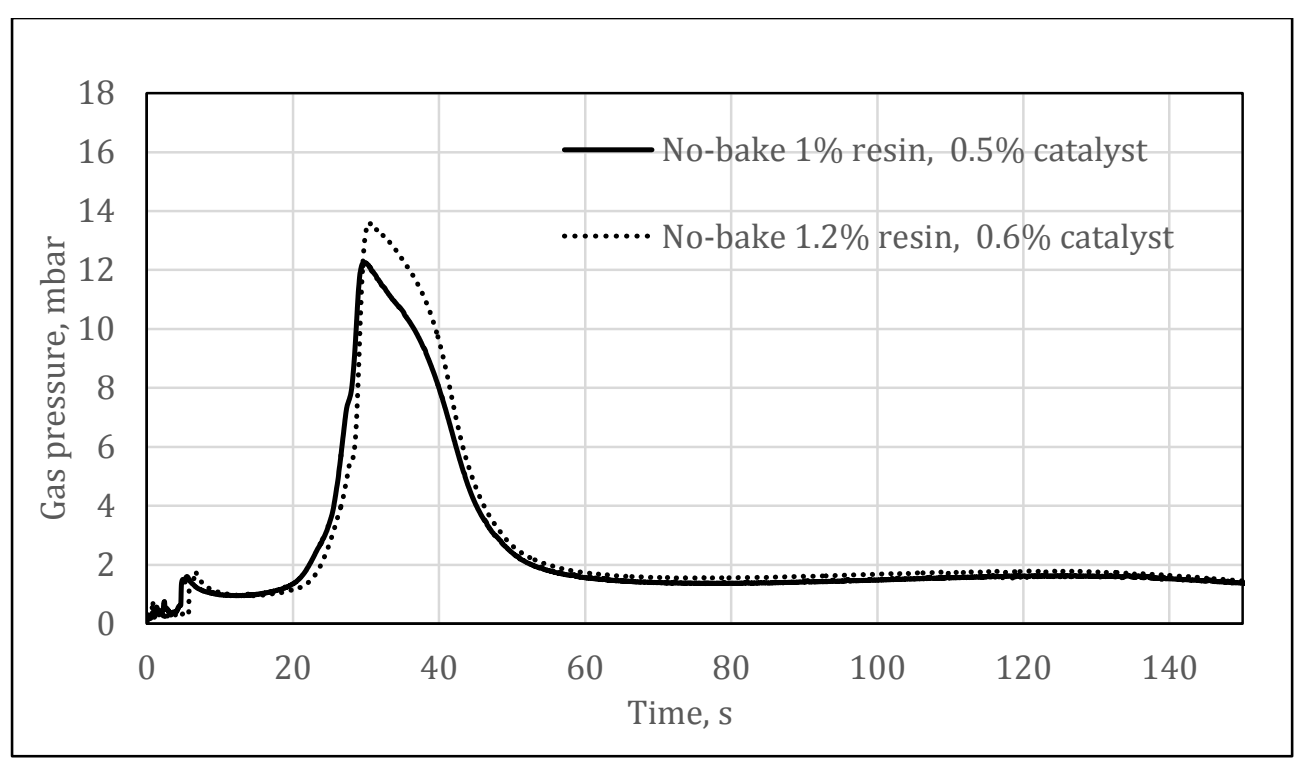

Figure 8 The effect of resin quantity on the pressure of the released gases in case of no-bake phenol

In the case of phenolic no-bake cores, core gas pressure does not increase significantly, and gas pressure peaks appear later in time. 


\section{Conclusion}

Casting defects caused by gas penetration are formed when the maximum gas pressure occurs a few seconds after casting, before the development of a solid skin. The characteristics of the gas pressure curves are significantly influenced by the resin quality. The effect of the amount of resin on the pressure may be different for different binder systems. Therefore, it is necessary to examine the different resin quantity of the cores of different qualities. The pressure peak values vary between 4-14 mbar in case of the different resin qualities.

\section{Acknowledgments}

The research was also supported BY the ÚNKP-17-3 New National Excellence Program of the Ministry of Human Capacities.

\section{References}

[1] Pattabhi, A.M. Lane, T.S. Piwonka (1975), Cast Iron Penetration on Sand Molds, Part III: Measurement of Mold-Metal Interfacial Gas Composition- AFS Trans. 96-208, 1259- 1264

[2] L. Winardi, H.E. Littleton, R.D. Griffin J.A. Griffin (2008) Gas from Green Sand Molds and Vapor Transport Zone- AFS Transactions p.381-399.

[3] IKO-Erbslöh, Jörg Baier, Martin Köpper (1994) Incidence and avoidance of defects attributable to molding sands - Manual of Casting Defects, P: 51-55

[4] Doru Michael Stefanescu (2017), Surface Quality, Penetration Defects and Casting Skin- Course Metallurgy, solidification and modeling of cast iron castings 5th edition, Presentation, Jönköping, Sweden

[5] L. Winard, H.E. Littleton, C.E. Bates (2007), Gas Pressure in Sand Cores- AFS Trans., 07-062(04), 303-312

[6] L. Tóth (1997), Gas evolution in foundry molding and core sand mixtures. Relationship of active bentonite and water content of bentonite molding compounds to plasticity and crack strength Thesis of Ph.D. Dissertation

[7] Louis Pilato (2010), Phenolic Resins: A Century of Progress- Springer, 451-502

[8] P.F. Ambidge, P.D.E. Biggins (1984), Environmental Problems Arising from the Use of Chemicals in Molding Materials- Developments for Future Foundry Prosperity p. 219.

[9] S.Ravi, J. Thiel (2017), Prediction of Core Gas Pressure from Chemically Bonded Sand Molds Using Process Simulation Software- AFS Transactions, 17-097 v125

[10] A. Chojecki, J. Mocek (2011), Gas pressure in sand mold poured with cast iron- Archives of Foundry Engineering Volume 11, Issue, 1/2011, 9-14

[11] L. Mádi, L. Varga (2018), The effect of gas permeability on the pressure of artificial resin-bonded core gases- IOP Conf. Ser.: Mater. Sci. Eng. 42601203 